\title{
Path Integration for Velocity-Dependent Potentials
}

\author{
Bruce Nelson ${ }^{1}$ and Benny Sheeks ${ }^{2}$ \\ 1 Department of Physics, University of Utah, Salt Lake City, UT 84112, USA \\ 2 Lincoln Laboratory, Massachusetts Institu'te of Technology, Lexington, MA 02173, USA
}

\begin{abstract}
We extent the prodistribution definition of path integrals to include Lagrangians with velocity-dependent potentials. We use Cameron-Martin transformations to evaluate a large part of a path integral exactly and give techniques for evaluating the remaining terms of the semi-classical expansion of the path integral. The Fredholm determinants, associated with these transformations, are evaluated explicitly in terms of Jacobi matrices defined by the classical system.
\end{abstract}

\section{Introduction}

We develop a method for computing the semi-classical expansion of path integrals for systems having velocity-dependent potentials. Our starting point is the definition of path integrals in terms of prodistributions that was introduced by DeWitt-Morette [1-3]. We extend her methods to include velocity-dependent potentials.

In the semi-classical expansion, the terms in the path integral for a wave function are expanded in a Taylor series around an appropriate classical path. The terms involving the second variation of the action can be combined with the Wiener prodistribution, that defines the path integral, to give a new Gaussian prodistribution that is adapted to integrating the higher order terms of the semiclassical expansion. The use of the new Gaussian prodistribution - or equivalently, the expansion around a classical trajectory instead of a straight line - has the advantage that a larger part of the path integral is evaluated exactly and hence the semi-classical expansion seems to converge faster than the Born expansion. The semi-classical expansion also brings out the connection between the quantum wave function and the family of classical trajectories.

The basic theory of Gaussian prodistributions and their semi-classical expansion is presented in [4]. We extend the results presented there to systems having velocity-dependent potentials. This paper is self-contained; however, many of the results that are derived in [4] are stated here without proof. 


\section{Classical Mechanics}

Electrodynamics is the classical example of a system that contains a velocitydependent potential. Because the potential is linear in the velocity, the quantization of the system using path integrals is straightforward. We define these path integrals in terms of projective systems of distributions introduced by DeWittMorette. This definition lends itself immediately to the semi-classical expansion of the quantum propagator $K$, in which $K$ can be computed to any desired order of $\hbar$ in terms of ordinary integrals on $R^{n}$. The Green's functions of the classical small disturbance operator will determine the prodistributions that are used in computing $K$. For this reason we first give a discussion of the Jacobi fields of the classical system and discuss some of their properties that will be important for computing and interpreting the path integrals.

A non-relativistic particle in an external electromagnetic field can be described by the Lagrangian

$$
L(q, \dot{q}, t)=\frac{m}{2} \dot{q}^{2}+e A \cdot \dot{q}-e V
$$

(we take units such that $c=1$ ). The action of the path $q$ (defined for the time interval $\left.T=\left[t_{a}, t_{b}\right]\right)$ is

$$
S[q]=\int_{T} L(q(t), \dot{q}(t), t) d t
$$

In order to compute the first and second variations of $S$, consider a one-parameter family of paths, $q(u, t)$. For each $u, q(u, \cdot)$ is a path. In flat space in Cartesian coordinates, for example, a convenient family is

$$
q(u, t)=Q(t)+u x(t)
$$

where $Q$ is the classical path and $x$ is a vector field along $Q$, representing the departure of $q$ from $Q$. In general, if

$$
x \equiv \frac{\partial q}{\partial u},
$$

then the first variation of $S$ is a linear function(al) of the field $x$ :

$$
\left.S^{\prime}(x) \equiv \frac{\partial S}{\partial u}\right|_{u=0}
$$

and the second variation is a bi-linear function of $x$ :

$$
S^{\prime \prime}(x, x)=\left.\frac{\partial^{2} S}{\partial u^{2}}\right|_{u=0} .
$$

If a two parameter family $q(u, v ; t)$ is considered, then $S^{\prime \prime}$ can be defined more generally as

$$
S^{\prime \prime}(x, y)=\left.\frac{\partial^{2} S}{\partial u \partial v}\right|_{u=v=0},
$$


where $x$ is as before and

$$
y \equiv \frac{\partial q}{\partial v} .
$$

$S^{\prime \prime}$ is then a symmetric, bi-linear form on the space of vector fields (with square integrable derivatives) along the path $Q \equiv q(\cdot, u=0, v=0)$. In general, we take $Q$ to be a classical path.

For the Lagrangian given by (1)

$$
\begin{aligned}
S^{\prime}[q](x)= & \left.\frac{m}{2} \dot{q} \cdot x\right|_{t_{a}} ^{t_{b}}+\left.e A \cdot x\right|_{t_{a}} ^{t_{b}} \\
& +\int_{t_{a}}^{t_{b}}\left(-m \ddot{q} \cdot x+e x \cdot \nabla A \cdot \dot{q}-e \dot{q} \cdot \nabla A \cdot x-e \frac{\partial A}{\partial t} \cdot x-e x \cdot \nabla V\right) d t .
\end{aligned}
$$

(We use juxtaposition with a dot to indicate contraction. Thus, for example, $x \cdot \nabla A$ $\cdot \dot{q} \equiv x^{\alpha} \nabla_{\alpha} A_{\beta} \dot{q}^{\beta}$. In terms such as $\dot{q} \cdot x$, a factor of $g$ is often left out: $\dot{q}^{\alpha} g_{\alpha \beta} x^{\beta}$.) The boundary terms arise from integrations by parts performed to eliminate the $\dot{x}$ terms. They are often neglected, but in fact will be important in the definition of the prodistributions used later. Use has also been made of the fact that

$$
\frac{d A}{d t}(q, t)=\frac{\partial A}{\partial t}+\dot{q} \cdot \nabla A
$$

$S^{\prime}$ can be written in terms of the magnetic field tensor $F$. Let

and define $F$ by

$$
\widetilde{(\nabla A})_{\alpha \beta} \equiv \nabla_{\beta} A_{\alpha} \text {, }
$$

$$
F \equiv \nabla A-\widetilde{\nabla A}
$$

Then

$$
\begin{aligned}
S^{\prime}[q](x)= & \left.\frac{m}{2} x \dot{q}\right|_{t_{a}} ^{t_{b}}+\left.e A \cdot x\right|_{t_{a}} ^{t_{b}} \\
& +\int_{t_{a}}^{t_{b}} x\left(-m \ddot{q}+e F \cdot \dot{q}-e \frac{\partial A}{\partial t}-e V V\right) d t .
\end{aligned}
$$

The classical path $Q$ is then given by $S^{\prime}[Q](x)=0$, for all $x$ such that $x\left(t_{a}\right)=x\left(t_{b}\right)=0$ and satisfies the Euler-Lagrange equations

$$
-m g \cdot \ddot{Q}+e F \cdot \dot{Q}-e \frac{\partial A}{\partial t}-e \nabla V=0 .
$$

The second variation of $S$ is

$$
\begin{aligned}
S^{\prime \prime}[Q](x, y)= & \left.m x \cdot y\right|_{t_{a}} ^{t_{b}}+\left.e x \cdot \widetilde{\nabla A} \cdot y\right|_{t_{a}} ^{t_{b}} \\
& +\int_{t_{a}}^{t_{b}}\left(-m x \cdot \ddot{y}+e x \cdot F \cdot \dot{y}+e x \cdot \widetilde{\nabla F \cdot \dot{Q}} \cdot y-e x \cdot \frac{\partial}{\partial t} \nabla A \cdot y-e x \cdot \nabla \nabla V \cdot y\right) d t,
\end{aligned}
$$

where all functions are to be evaluated along the classical path $Q$. The integral can be written

$$
m \int x \cdot\left(\mathscr{J}_{t} \cdot y\right) d t
$$


where $\mathscr{J}_{t}$ is the Jacobi operator:

$$
\mathscr{J}_{t}=-g \nabla_{t}^{2}+\frac{e}{m} F \nabla_{t}+\frac{e}{m} \widetilde{\nabla F \cdot \dot{Q}}-\frac{e}{m} \frac{\partial}{\partial t} \widetilde{\nabla A}-\frac{e}{m} \nabla \nabla V .
$$

The operator $S^{\prime \prime}[Q]$ is a symmetric, bi-linear form on the space of vector fields along $Q$; i.e.

and

$$
S^{\prime \prime}(x, y)=S^{\prime \prime}(y, x)
$$

$$
S^{\prime \prime}(x+y, z)=S^{\prime \prime}(x, z)+S^{\prime \prime}(y, z) .
$$

This property of $S^{\prime \prime}$ will be important for defining a generalized Gaussian prodistribution that "incorporates" the quadratic terms in the semi-classical expansion of the Lagrangian.

The covariance of the new Gaussian prodistribution will be an elementary kernel of the Jacobi operator. It can be constructed from the Jacobi matrices of $\mathscr{J}_{t}$. Further, these Jacobi matrices will appear as weight factors in the path integrals for the propagator and for a general wave function. These weight factors can be interpreted in terms of families of classical paths. For these reasons we discuss some of the properties of these Jacobi fields.

Let $Q$ be a classical path. A vector field $h$ along $Q$ is a Jacobi field if $\mathscr{J}_{t} h(t)=0$. If $h$ is such that $h\left(t_{a}\right)=0$, then it can be written in terms of a two point tensor - a Jacobi matrix $-J$ as

$$
h^{\alpha}(t)=J^{\alpha \beta}\left(t, t_{a}\right) g_{\beta \gamma}\left(t_{a}\right) \dot{h}^{\alpha}\left(t=t_{a}\right),
$$

where $J$ is defined by

$$
\begin{aligned}
\mathscr{J}_{t} J\left(t, t_{a}\right) & =0, \\
J\left(t_{a}, t_{a}\right) & =0, \\
\nabla_{t} J(t & \left.=t_{a}, t_{a}\right)=g^{-1}\left(t_{a}\right) .
\end{aligned}
$$

Similarly any Jacobi field $k$ along $Q$ with $\dot{k}_{(}\left(t_{a}\right)=0$ can be written in terms of Jacobi matrix $\bar{K}$ as

$$
k^{\alpha}(t)=\bar{K}^{\alpha \beta}\left(t, t_{a}\right) g_{\beta \gamma}\left(t_{a}\right) k^{\gamma}\left(t_{a}\right)
$$

where $\bar{K}$ is defined by

$$
\begin{aligned}
\mathscr{J}_{t} \bar{K}\left(t, t_{a}\right) & =0, \\
\bar{K}\left(t_{a}, t_{a}\right) & =g^{-1}\left(t_{a}\right), \\
\nabla_{t} \bar{K}(t & \left.=t_{a}, t_{a}\right)=0 .
\end{aligned}
$$

Any Jacobi field can be written in terms of $J$ and $\bar{K}$. For later work, it is convenient to define a third Jacobi matrix $K$, which is linear combination of $J$ and $\bar{K}$ :

$$
\begin{aligned}
\mathscr{J}_{t} K\left(t, t_{a}\right) & =0 \\
K\left(t_{a}, t_{a}\right) & =g^{-1}\left(t_{a}\right), \\
\nabla_{t} K\left(t=t_{a}, t_{a}\right) & =\frac{-e}{m} g^{-1}\left(t_{a}\right) \cdot \widetilde{\nabla A}\left(t_{a}\right) \cdot g^{-1}\left(t_{a}\right) .
\end{aligned}
$$


(This Jacobi matrix should not be confused with the propagator, also denoted by K.)

If $N$ is the inverse of $K$ defined by

then the skew tensor

$$
K\left(t, t_{a}\right) N\left(t_{a}, t\right)=1
$$

$$
\omega_{\alpha \beta}(t) \equiv g_{\alpha \mu}(t) \dot{K}^{\mu \delta}\left(t, t_{a}\right) N_{\delta \beta}\left(t_{a}, t\right)-g_{\beta \mu}(t) \dot{K}^{\mu \delta}\left(t, t_{a}\right) N_{\delta \alpha}\left(t_{a}, t\right)
$$

represents the vorticity, or rate of rotation, of a family of classical paths whose initial orientation (at $t_{a}$ ) are determined by the boundary conditions on $K$ (see [5, p. 83]). For the particular $K$ defined here,

$$
\omega_{\alpha \beta}(t)=\frac{e}{m} F_{\alpha \beta}(Q(t)) .
$$

(We prove this result in the appendix.) This equation relates the spiraling of a family of classical paths directly to the magnetic field.

Another interesting function of $K$ is its determinant $\sigma$ :

$$
\sigma(t)=\operatorname{det} K^{\alpha \beta}\left(t, t_{a}\right) .
$$

It represents the ratio of the infinitesimal volume $V$ swept out by a tube of classical paths at $t$ to the volume at $t_{a}$ :

Fig. 1.

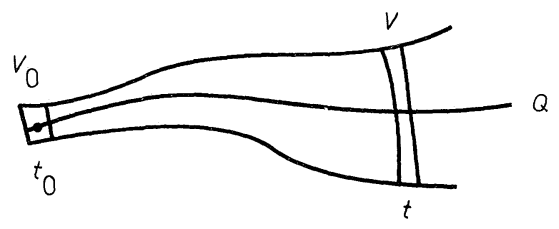

$$
\sigma=\frac{v}{v_{0}}
$$

Alternately, if the classical flow is looked at as a map of the configuration space onto itself (for each $t$ ) then $K$ will be the derivative of this transformation and $\sigma$ will be the Jacobian of the transformation.

The Green's function $G_{+}$of $\mathscr{J}_{t}$ with boundary conditions

$$
\begin{aligned}
& G_{+}\left(t=t_{a}, s\right)=0, \\
& \nabla_{t} G\left(t=t_{a}, s\right)=-e g^{-1}\left(t_{a}\right) \cdot(\nabla A)\left(t_{a}\right) \cdot G\left(t_{a}, s\right)
\end{aligned}
$$

[these boundary conditions will guarantee that $G$ is symmetric:

$$
\left.G_{+}^{\sim}(t, s)=G_{+}(s, t)\right]
$$

satisfying

$$
\mathscr{J}_{t} G_{+}(t, s)=1 \cdot \delta(t-s)
$$

can be written in terms of $J, K$, and $N$ as

$$
G_{+}(t, s)=\theta(t-s) K\left(r, t_{a}\right) \cdot N\left(t_{a}, t_{b}\right) \cdot J\left(t_{b}, s\right)-\theta(s-t) J\left(t, t_{b}\right) \cdot \tilde{N}\left(t_{b}, t_{a}\right) \cdot \tilde{K}\left(t_{a}, s\right),
$$


where $\tilde{K}^{\alpha \beta}(t, s) \equiv K^{\beta \alpha}(s, t)$. This $G_{+}$will be used in defining the prodistribution for the semi-classical expansion for the wave function of the quantum system defined by the Lagrangian of Eq. (1). The fact that $G_{+}$can be written explicitly in terms of the step function and products of Jacobi matrices is very useful for simplifying certain path integrals.

These results can be expressed conveniently in terms of Poisson brackets. If the classical path $q$ is given in terms of $q\left(t_{a}\right)$ and $p\left(t_{a}\right)\left(\right.$ where $\left.p \equiv \frac{\partial L}{\partial \dot{q}}\right)$ then

$$
\begin{aligned}
J^{\alpha \beta}(t, s) & =-m\left[q^{\alpha}(t), q^{\beta}(t)\right] \\
& \equiv m \frac{\partial q^{\alpha}(t)}{\partial p_{\gamma}\left(t_{a}\right)} \cdot \frac{\partial q^{\beta}(s)}{\partial q^{\gamma}\left(t_{a}\right)}-m \frac{\partial q^{\alpha}(t)}{\partial q^{\gamma}\left(t_{a}\right)} \frac{\partial q^{\beta}(s)}{\partial p_{\gamma}\left(t_{a}\right)} .
\end{aligned}
$$

Similarly, $q$ can be defined in terms of $q\left(t_{b}\right)$ and $p\left(t_{b}\right)$. Then $J$ is given by the same expression with $t_{a}$ replaced by $t_{b}$. $K$ can likewise be written in terms of Poisson brackets. For $q=q\left(t, q\left(t_{a}\right), p\left(t_{a}\right)\right)$

$$
\begin{aligned}
K^{\alpha \beta}(t, s) & =\left[q^{\alpha}(t), p_{\gamma}(s)\right] g^{\gamma \beta}(s) \\
& \equiv \frac{\partial q^{\alpha}(t)}{\partial q^{\mu}\left(t_{a}\right)} \frac{\partial p_{\gamma}(s)}{\partial p_{\mu}\left(t_{a}\right)} g^{\gamma \beta}(s)-\frac{\partial q^{\alpha}(t)}{\partial p_{\mu}\left(t_{a}\right)} \frac{\partial p_{\gamma}(s)}{\partial q^{\mu}\left(t_{a}\right)} g^{\gamma \beta}(s) .
\end{aligned}
$$

Again, if $q$ is given in terms of $q\left(t_{b}\right)$ and $p\left(t_{b}\right)$, then $K$ will be given by the same expression with $t_{a}$ replaced by $t_{b}$.

The inverses $M$ and $N$ of $J$ and $K$ take a particularly convenient form. For example, if $q=q\left(t, q\left(t_{a}\right), p\left(t_{a}\right)\right)$, then

$$
M_{\alpha \beta}\left(t, t_{a}\right)=-\frac{1}{m} \frac{\partial p_{\beta}\left(t_{a}\right)}{\partial q^{\alpha}(t)}
$$

and

$$
N_{\alpha \beta}\left(t, t_{a}\right)=g_{\alpha \mu}(t) \frac{\partial p_{\beta}\left(t_{a}\right)}{\partial p_{\mu}(t)} .
$$

The proofs of these results and a discussion of their consequences are given in [6].

\section{The Path Integral and its Semi-Classical Expansion}

In the Schrödinger description of quantum mechanics, a classical system with a Hamiltonian $H$ is quantized by defining a wave function $\psi$ as a solution to the Schrödinger equation (formed from $H$ ) with a particular set of boundary conditions. In the path integral description, a wave function $\psi\left(b, t_{b}\right)$ is defined in terms of its value $\phi(a)$ at some initial time and a path integral that involves the classical Lagrangian. We define $\psi$ by the generalized Feynman-Kac formula:

$$
\psi\left(b, t_{b}\right)=\int_{X_{+}} d w_{+}^{w}(x) \exp \left(\frac{i}{\hbar} \int_{T}\left(L(q(t), \dot{q}(t), t)-L_{0}\right) d t\right) \phi\left(b+\mu x\left(t_{a}\right)\right) .
$$


Here $X_{+}$is the space of all continuous paths, parametrized by the time interval $T \equiv\left[t_{a}, t_{b}\right]$, that end at the origin (of the configuration space $R^{n}$ ) at $t_{b} . L$ is the Lagrangian of the system to be quantized. In terms of the integration variable $x, L$ is to be evaluated at

$$
q(t)=b+\mu x(t)
$$

where $\mu \equiv \sqrt{\hbar / m} . L_{0}$ is the free particle part of $L$ :

$$
L_{0}=\frac{1}{2} m \dot{q} \cdot g \cdot \dot{q} .
$$

The path integral is defined in terms of the Wiener prodistribution $d w_{+}^{w}(x)$.

Our path integral can be related to the one defined by Feynman in the following way. First, since we choose as the domain of integration the space $X_{+}$of paths that end at zero, it is necessary to shift all arguments by an amount $b$. The Wiener prodistribution can be thought of as representing the exponential of the free particle part of the Lagrangian plus the "measure" $\mathscr{D}(x)$ :

$$
\exp \left(\frac{i}{2} \int_{T} \dot{q}^{2} d t\right) \mathscr{D}(x)
$$

We choose the correspondence of $d w_{+}^{w}(x)$ to be for the kinetic energy of a system with "mass" $\hbar / m$. In order to recover the general case, it is necessary to scale the integration variable by $\sqrt{\hbar / m}$, hence the appearance of the factor $\mu$. It must be emphasized that this correspondence is strictly formal; it is offered here only to make the Feynman-Kac formula plausible. We take it as a definition of $\psi$. The actual relationship between the prodistribution path integral and Feynman's is discussed below. (The Feynman-Kac formula has been derived from first principles for the case of velocity-independent potentials [4, Sect. 2.3].) Here $\psi$, defined by the generalized Feynman-Kac formula, satisfies the expected Schrödinger equation.

If $L$ corresponds to a system with a velocity dependent potential $\dot{q} \cdot A$, then the path integral will contain the expression

$$
\int_{T} \dot{x} \cdot A d t
$$

This is a purely formal expression. The reason is that as we have defined the path integral, $x$ is a stochastic variable and it does not have a derivative $\dot{x}$ at any point (for almost all $x$ ). For this reason, we define any expression involving $\dot{x}$ in this way as

$$
\int_{T} d x(t) \cdot A
$$

The differential $d x(t)$ is a stochastic differential. Giving meaning to expressions involving $d x$ is the subject of stochastic calculus.

In order to compute the path integral (13) we will expand all functions around a suitable classical path $Q$. This will correspond to a Taylor expansion in $\mu$. Using

$$
\hbar=m \mu^{2} \text {, }
$$


the expansion will have terms beginning at order $\mu^{-2}$. The terms of this order will be independent of $x$ and can be taken out of the integral. The terms of order $\mu^{-1}$ will cancel as a consequence of $Q$ satisfying the Euler-Lagrange equations. The terms of order $\mu^{0}$ will be quadratic in $x$ and can be combined with $d w_{+}^{w}(x)$ to give a new Gaussian prodistribution $d \bar{w}_{+}(x)$. The remaining terms can be combined in groups of the same power of $\mu$ and can be explicitly integrated using $d \bar{w}_{+}(x)$. Stopping with the $\mu^{0}$ terms constitutes the WKB approximation. The calculation of the higher order terms constitutes the semi-classical expansion.

In order that the path integral can be represented by a semi-classical expansion, it is necessary to choose a particular classical path about which to expand. There are several cases in which there is a natural classical path $Q$. First, if $\phi$ is a $\delta$-function, say $\delta\left(q\left(t_{a}\right)-a\right)$, then $\psi$ will be the propagator $K\left(b, t_{b} ; a, t_{a}\right)$ and $Q$ will be the classical path between $a$ at $t_{a}$ and $b$ at $t_{b}$. (We will assume for the present that $Q$ is unique. When it is not, the analysis presented here can be immediately generalized, using the techniques presented in [4, p. 313].) Second, if $\phi$ is defined in terms of some momentum $p$, as for example in an initial plane wave state, there $Q$ will be the classical path which has momentum equal to $p$ at $t_{a}$ and which passes through $b$ at $t_{b}$. We will consider the first case in detail here and afterwards will present the results for the case when $\phi$ is a generalized plane wave.

The propagator $K$ is then given by

$$
\begin{aligned}
K\left(b, t_{b} ; a, t_{a}\right)= & \int_{X_{+}} d w_{+}^{w}(x) \exp \left(\frac{i}{\hbar} \int_{t_{a}}^{t_{b}}(e A(b+\mu x(t)) \cdot \mu d x(t)-e V(b+\mu x(t)) d t)\right. \\
& \cdot \delta\left(b+\mu x\left(t_{a}\right)-a\right) .
\end{aligned}
$$

Let now $Q$ be the classical path passing through $a$ at $t_{a}$ and through $b$ at $t_{b}$ :

$$
\begin{gathered}
+m g_{\alpha \beta} \ddot{Q}^{\beta}+e F_{\alpha \beta} \dot{Q}^{\beta}-e \frac{\partial A_{\alpha}}{\partial t}-e \nabla_{\alpha} V=0, \\
Q\left(t_{a}\right)=a, \\
Q\left(t_{b}\right)=b .
\end{gathered}
$$

The steps in the semi-classical expansion are essentially the same as those described on in Sect. 3.4 of [4]. We outline the procedure here and present the results. First the integration variable is changed from $x$ to $y$, where $y$ represents the departure of an arbitrary path $q$ from the classical path. Thus

$$
q=b+\mu x \mapsto Q+\mu y .
$$

Under this change of variables, the Wiener prodistribution $d w_{+}^{w}(x)$ is mapped into the Wiener prodistribution of $y$ with a phase factor:

$$
d w_{+}^{w}(x)=d w_{+}^{w}(y) \exp \left(\frac{i}{2} \int_{t_{a}}^{t_{b}} \frac{1}{\mu^{2}} \dot{Q}^{2}(t) d t+i \int_{t_{a}}^{t_{b}} \frac{1}{\mu} \dot{Q}(t) \cdot d x(t)\right) .
$$

The stochastic integral can be simplified by an integration by parts. Then $d w_{+}$is

$$
d w_{+}^{w}(x)=d w_{+}^{w}(y) \exp \left(\frac{i}{\hbar} \int \frac{m}{2} \dot{Q}^{2} d t-\frac{i}{\mu} \dot{Q} \cdot x\left(t_{a}\right)-\frac{i}{\mu} \int \ddot{Q} \cdot x d t+\frac{i}{\mu} \dot{Q} \cdot x\left(t_{b}\right)\right) .
$$


The terms in the exponent in the path integral for $K(14)$ are now functions of $Q+\mu y$, and can be expanded in powers of $\mu$, i.e. they can be expanded in a Taylor series about the classical path. The expansion for $A$ needs to be done carefully, since it involves stochastic integrals. The result is

$$
\begin{aligned}
K\left(b, t_{b} ; a, t_{a}\right)= & \exp \left(\frac{i}{\hbar} \bar{S}(B, A)\right) \int_{Y_{+}} d w_{+}^{w}(y) \exp \left(\frac{i}{2} \int_{T} H(y, d y, Q)\right) \\
& \cdot \exp \left(i \int_{T} \Omega(y, Q, d y)\right) \delta\left(\mu y\left(t_{a}\right)\right) .
\end{aligned}
$$

$\bar{S}$ is the classical action along $Q$ from $\left(b, t_{b}\right)$ to $\left(a, t_{a}\right)$ :

$$
\bar{S}(B, A)=\int_{t_{a}}^{t_{b}}\left(\frac{m}{2} \dot{Q}^{2}+e A \cdot \dot{Q}-e V\right) d t .
$$

It comes from the terms in the path integral that are not functions of $y$. $H$ represents the terms linear and quadratic in $y$ that come from the measure change and from the Taylor expansion:

$$
\begin{aligned}
\frac{1}{2} \int_{T} H(y, d y, Q) \equiv & \frac{1}{\mu}\left(\frac{-1}{m} A\left(t_{a}\right) \cdot y\left(t_{a}\right)+\int_{T}\left(-\ddot{Q} \cdot y-\frac{1}{m} \dot{Q} \cdot F \cdot y-\frac{1}{m} \nabla V \cdot y\right) d t\right) \\
& +\frac{1}{2 m} \int_{T}\left(y \cdot F \cdot d y+y \cdot \widetilde{\nabla F \cdot \dot{Q}} \cdot y d t-y \cdot \frac{\partial}{\partial t}(\widetilde{\nabla A}) \cdot y d t-y \cdot \nabla \nabla V \cdot y d t\right) \\
& -\frac{1}{2 m}(y \cdot \widetilde{\nabla A} \cdot y)\left(t_{a}\right)
\end{aligned}
$$

(where use has been made of the fact that $\frac{\mu}{\hbar}=\frac{1}{\mu \mathrm{m}}$ ). The $\frac{1}{\mu}$ term is zero since $Q$ satisfies the Euler-Lagrange equations; and

$$
\int_{Y_{+}} d w_{+}^{w}(y) e^{F\left(y\left(t_{a}\right)\right)} \delta\left(y\left(t_{a}\right)\right)=e^{0}=1 .
$$

[For the more general initial wave function discussed below, the $1 / \mu$ terms will again cancel. The quadratic boundary term will not be zero. We choose, therefore, to retain the $(y \cdot \overparen{\nabla A} \cdot y)\left(t_{a}\right)$ term here in order to make the generalization from the $\delta$-function more direct.]

The $\Omega$ term contains all the terms cubic and higher in the Taylor expansions of $A \cdot \dot{q}$ and of $V$. Note that each term in $\Omega$ is at most linear in $d y$, so that the expression makes sense; that is, there are no terms that contain $\int d y d y$ or, even worse, $\int \dot{y}^{3} d t$. The propagator is then

$$
\begin{aligned}
K\left(b, t_{b} ; a, t_{a}\right)= & \exp \left(\frac{i}{\hbar} \bar{S}(B, A)\right) \int_{Y_{+}} d w_{+}^{w}(y) \exp \left[\frac{i}{2 m}(y \cdot \widetilde{\nabla A} \cdot y)\left(t_{a}\right)\right. \\
& \left.+\frac{i}{2 m} \int_{T}\left(y \cdot F \cdot d y+y \cdot \widetilde{\nabla F \cdot \dot{Q}} \cdot y d t-y \cdot \frac{\partial}{\partial t}(\widetilde{\nabla A}) \cdot y d t\right)\right] \\
& \cdot \exp \left(i \int_{T} \Omega(y, d y, Q, \dot{Q}, d t)\right) \delta\left(\mu y\left(t_{a}\right)\right) .
\end{aligned}
$$


Notice that if $d y(t)$ is formally written as $\dot{y}(t) d t$, then the quadratic term is essentially $S^{\prime \prime}(y, y)$, aside from a $\ddot{y}$ term:

$$
\int_{T} y(t) \cdot\left(\mathscr{J}_{t} \cdot y(t)\right) d t-\int_{T}\left(-y(t) \cdot \nabla_{t}^{2} \cdot y(t) d t\right) .
$$

This suggests that the quadratic exponential can be combined with the Wiener measure to give a "larger" Gaussian measure.

\section{The Cameron-Martin Transformation (see [4] and [7])}

We now describe how the quadratic part of the semi-classical expansion is combined with the Wiener prodistribution to give a larger Gaussian prodistribution, which can then be used to integrate the terms in a power expansion of the remaining terms in the semi-classical expansion. The advantage of this procedure is that all of the information about the classical system is incorporated into the measure and the quantum corrections come then in the natural powers of $\hbar$. This corresponds to doing an expansion around the classical system rather than around the free system. In general, such expansions converge faster; at any rate they offer the conceptual advantage of showing how the quantum effects can be sewn onto the classical framework.

In order to motivate the use of Fourier transforms to describe Gaussian prodistributions in the infinite-dimensional path spaces, we mention a few properties, familiar from the finite dimensional case. A normalized Gaussian measure can be written in terms of some square matrix $A$ as

$$
d \gamma(x)=d x^{1} \ldots d x^{n}|\operatorname{det} A|^{1 / 2} \exp \left(\frac{i}{2} x \cdot A \cdot x\right) .
$$

Its Fourier transform takes the much simpler form

$$
(\mathscr{F} \gamma)(y)=\exp \left(-\frac{i}{2} y \cdot A^{-1} \cdot y\right)
$$

where $A^{-1}$ is the inverse of $A$. Notice that $\mathscr{F} \gamma$ does not contain the volume factor $d x^{1} \ldots d x^{n}$ or the normalization factor $|A|^{1 / 2}$. This fact makes $\mathscr{F} \gamma$ the natural object to use in extending Gaussian measures to infinite-dimensional spaces. Also, even in the finite-dimensional case, the fact that $\mathscr{F} \gamma$ does not contain the volume factor makes its transformation under coordinate transformations much simpler to study than that of $\gamma$. Next, if $d \gamma$ is multiplied by a Gaussian factor $e^{\frac{i}{2} x \cdot B \cdot x}$, the combination can be written as a new Gaussian measure $d \bar{\gamma}$ :

$$
d \gamma(x) \exp \left(\frac{i}{2} x \cdot B \cdot x\right) \sim d \bar{\gamma}(\chi)
$$

where the covariance of $\bar{\gamma}$ will be just $A+B$. However, if $\bar{\gamma}$ is taken to be a normalized measure, the connection in (16) will not be equality, only proportionality, since the normalization factor in $d \gamma$ is just $|A|^{1 / 2}$ and not $|A+B|^{1 / 2}$. Thus

$$
d \gamma(x) \exp \left(\frac{i}{2} x \cdot B \cdot x\right)=D d \bar{\gamma}(x)
$$


where $D$ is some determinant, determined by $A$ and $B$. This is the result that we want to use in the semi-classical expansion.

The Wiener prodistribution $d w_{+}(x)$ is like Feynman's

$$
\mathscr{D} x \exp \left(\frac{i}{2} \int \dot{x}^{2} d t\right)
$$

In order to keep the analogy with the finite-dimensional case clear, we can extend the summation convention to include integration over repeated time variables. Then

$$
d w_{+}(x) \sim \mathscr{D} x \exp \left(\frac{i}{2} \dot{x} \cdot \dot{x}\right) .
$$

The fact that $\dot{x}$, instead of $x$, appears is a little troubling. The appearance of the finite-dimensional case can be recovered if we perform an integration by parts:

$$
d w_{+}(x) \sim \mathscr{D} x \exp \left(\frac{i}{2}(x \cdot \mathscr{J} \cdot x+x(0) \cdot \dot{x}(0))\right) .
$$

Now instead of the matrix $A$ multiplying $x$, we have the operator

$$
\mathscr{J} \equiv-\frac{d^{2}}{d t^{2}}
$$

on $x$. The boundary terms assure that the exponent is symmetric. The Fourier transform of $w_{+}$will be just the exponential of the inverse of $\mathscr{J}$. The inverse of an operator is a Green's function $G$. Again we choose the boundary conditions on $G$ so that it is symmetric.

In the semi-classical expandion $d w_{+}$is multiplied by the exponential of a quadratic expression. These two can be combined to give a new prodistribution $d \bar{w}_{+}$. There will in general be a normalization factor $D$ that appears. In particular, we have

$$
d w_{+}(y) \exp \left(\frac{i}{2} y \cdot H \cdot y\right)
$$

where $H$ is the operator

$$
H(t)=\frac{e}{m}\left(F \frac{d}{d t}+\nabla F \cdot \dot{Q}-\frac{\partial}{\partial t} \nabla A\right)
$$

(and the dots represent not only index summation but also integration). Now, $H$ plus $\mathscr{J}$ from $d w_{+}$is just the Jacobi operator $\overline{\mathscr{J}}$ for a particle in an electromagnetic field [given by (5)]. Thus, the covariance $\bar{G}$ of $\bar{w}_{+}$is just the Green's function given by (11) or (12). As noted, the boundary conditions assume that $\bar{G}$ is symmetric. Thus we can write

$$
d w_{+}(y) \exp \left(i \int \frac{e}{m}\left(y \cdot F \cdot d y+y \cdot\left(\nabla F \cdot \dot{Q} \cdot-\frac{\partial}{\partial t} \nabla A\right) \cdot y d t\right)\right)=D d w_{+}(y),
$$

where $\bar{w}_{+}$is the Gaussian prodistribution defined by the covariance $\bar{G}$. This is the result we wanted. 
At this point, one might worry that since the factor $D$ is an infinite-dimensional determinant, it might well be infinite. However we have shown in [8] that not only is $D$ finite, but it can be written as the finite-dimensional determinant of a Jacobi field:

$$
D=\left|\operatorname{det} K\left(t_{b}, t_{a}\right)\right|^{-1 / 2},
$$

where $K$ is the Jacobi matrix defined by (8).

To compute the higher order terms in the semi-classical expansion, one expands the factor $e^{i f \Omega}$ in a power series. (The expression $\Omega$ is itself a power series in $\hbar$.) Terms of the same order in $\hbar$ are combined and evaluated using the prodistribution $d \bar{w}_{+}$. A complete discussion of the semi-classical expansion along with many examples of the explicit evaluation of path integrals can be found in [4].

We note here that a derivative term in an integral, such as

$$
\int d \bar{w}_{+}(y) \int y(t) \cdot F(t) \cdot \dot{y}(t) d t
$$

can be defined by

$$
\int d t \frac{d}{d s}\left(\int d \bar{w}_{+}(y) y(t) F(t) y(s)\right)_{s=t} .
$$

This formal result can be justified by using the symmetry properties of the original Wiener prodistribution coupled with those of the Cameron-Martin transformation that is associated with the change of prodistributions $d \bar{w}_{w} \rightarrow d w_{+}$.

\section{The Path Integral for a Wave Function with a "Momentum" Initial Value}

The propagator $K$ lends itself to a semi-classical expansion because in general there will be a well-defined classical path between the two endpoints. Another path integral that has a natural semi-classical expansion is the Feynman-Kac formula when the initial wave function is a generalized momentum state.

If at some time $t_{a}$ a function $S_{0}$ is given on the configuration space $M$, then its gradient $\nabla S_{0}$ will define a vector field. This vector field will define at each point a classical trajector (passing through the point at $t_{a}$ with velocity $\nabla S_{0}$ ). For each time after $t_{a}$, this family of trajectories will define a map of $M$ into itself. For how long this map remains surjective depends on the geometry of $M$, on the strength and nature of the potentials defining the classical system, and on how "flat" the equipotential surfaces of $S_{0}$ are (or equivalently, how "parallel" the initial velocity vectors $\nabla S_{0}$ are).

For an initial wave function $\phi$ defined in terms of $S_{0}$ by

$$
\phi(x)=T(x) e^{i S_{0}(x) / \hbar}
$$

(where the only $\hbar$ dependence is that explicitly shown) there will be a natural semiclassical expansion for the wave function $\psi\left(b, t_{b}\right)$ at a later time $t_{b}$. For the case of a particle in an external electromagnetic field (on $R^{n}$ ) the Feynman-Kac formula for $\psi$ is

$$
\psi\left(b, t_{b}\right)=\int_{X_{+}} d w_{+}^{w}(x) \exp \left(\frac{i}{\hbar} \int_{t_{a}}^{t_{b}}(e A(q(t)) \cdot \dot{q}(t)-e V(q(t))) d t\right) \phi\left(q\left(t_{a}\right)\right),
$$


where $q$ is to be written in terms of $x$ as

$$
q(t)=b+\mu x(t) .
$$

For a short enough time after $t_{a}$, the path integral can be expanded around the classical path $Q$ defined by

$$
\begin{gathered}
Q\left(t_{b}\right)=b, \\
\dot{Q}\left(t_{a}\right)=\frac{1}{m} \nabla S_{0}(a) .
\end{gathered}
$$

The point $a \equiv Q\left(t_{a}\right)$ is defined to be that point through which the classical path $Q$ passes at $t_{a}$ having velocity $\frac{1}{m} \nabla S_{0}(a)$ and passing through $b$ at $t_{b}$. We assume that such a point $a$ exists and that it is unique. For the semi-classical expansion, the integral in the exponential, $T$, and $S_{0}$ are expanded around $Q$. Terms up to $\mu^{0}$ (quadratic in the path integral variable) will define the WKB approximation and the enlarged Gaussian prodistribution with respect to which the remaining terms in the semi-classical expansion can be integrated. The result is that

$$
\begin{aligned}
\psi\left(b, t_{b}\right)= & \left|\operatorname{det} K\left(t_{b}, t_{a}\right)\right|^{-1 / 2} \int_{X_{+}} d \bar{w}_{+}(y) \exp (i \Omega(Q, y, d y, t, \mu)) \\
& \cdot\left(T(a)+\mu y \nabla T(a)+\frac{\mu^{2} y^{2}}{2} \nabla^{2} T(a)+\ldots\right),
\end{aligned}
$$

where $K$ is a Jacobi matrix, similar to the one defined in the previous section, except that its boundary conditions are

$$
\begin{aligned}
K^{\alpha \beta}\left(t_{a}, t_{a}\right) & =g^{\alpha \beta}\left(t_{a}\right), \\
\nabla_{t} K^{\alpha \beta}\left(t=t_{a}, t_{a}\right) & =g^{\alpha \mu}\left(t_{a}\right)\left(\nabla_{\mu} \nabla_{v} S_{0}(a)-e \nabla_{v} A_{\mu}(a)\right) g^{v \beta}\left(t_{a}\right)
\end{aligned}
$$

and $d \bar{w}_{+}$has as its covariance

$$
G_{+}(t, s)=\theta(s-t) K\left(t, t_{a}\right) N\left(t_{a}, t_{b}\right) J\left(t_{b}, s\right)-\theta(t-s) J\left(t, t_{b}\right) \tilde{N}\left(t_{b}, t_{a}\right) \tilde{K}\left(t_{a}, s\right) .
$$

( $J$ is defined the same as before.) $G_{+}$is an elementary kernel of the Jacobi operator with the boundary conditions

$$
\begin{gathered}
G_{+}\left(t_{b}, s\right)=0, \\
\nabla_{t} G_{+}^{\mu \nu}\left(t_{a}, s\right)=g^{\mu \alpha}\left(t_{a}\right)\left(\frac{1}{m} \nabla_{\alpha} \nabla_{\beta} S_{0}(a)-\frac{e}{m} \nabla_{\beta} A_{\alpha}(a)\right) G_{+}^{\beta v}\left(t_{a}, s\right) .
\end{gathered}
$$

The presence of $|\operatorname{det} K|^{-1 / 2}$ in the solution for $\psi$ shows the strong dependence of the WKB approximation on the behavior of classical paths. If the paths are diverging

Fig. 2

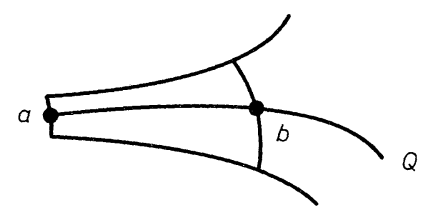


from $a$, then the effect of a second-order neighborhood of classical paths around a are spread out. Their effect at $b$ is lessened. This is shown by $\operatorname{det} K>1$. Since $\operatorname{det} K$ represents the relative volumes of the paths at $b$ compared to $a$, it is greater than one. However $\psi$ contains $|\operatorname{det} K|^{-1 / 2}$. As the paths spread out, the influence from $a$ is weakened. Similarly, if the paths converge, $|\operatorname{det} K|<1$, and $|\operatorname{det} K|^{-1 / 2}>1$. The effects of the paths from $a$ is concentrated at $b$.

If the paths actually cross, $a$ and $b$ are conjugate, $|\operatorname{det} K|^{-1 / 2}=\infty$, and the WKB approximation is no longer valid. It might appear that the whole semiclassical expansion breaks down at conjugate points. However, a careful analysis [4, p. 313] shows that as long as the path $Q$ can be defined, a calculation of the higher order terms in the semi-classical expansion show it to be valid on and beyond conjugate points.

It is worth noting that a change of gauge $A \rightarrow \bar{A}=A+\nabla \phi$ does not affect the Euler-Lagrange or Jacobi equations but does change the boundary conditons on $K$, and more importantly, on $Q$ itself. Thus in the Feynman-Kac formula (21) it is obvious that a gauge transformation introduces only a phase factor. However, once the semi-classical expansion is done, the particular gauge choice is intimately involved in the results. It is possible to define path integrals in the $U^{1}$ principle fibre bundle over the configuration space and thus be able to quantize an electrodynamic system in a gauge invariant way. This approach is presented in [9].

\section{Appendix}

We would like to establish the relationship between the vorticity of a particular family of classical paths and the magnetic field (10). In particular, if $Q$ is a classical path and $K$ is a Jacobi matrix along $Q$ satisfying the initial conditions given by (8), then the vorticity, defined by (9) is given simply by

$$
\omega_{\alpha \beta}(t)=\frac{e}{m} F_{\alpha \beta}(Q(t)) .
$$

Thus, for

$$
\begin{gathered}
\mathscr{J}_{t} K(t)=0, \\
K\left(t_{a}\right)=g^{-1}\left(t_{a}\right),
\end{gathered}
$$

and

$$
\dot{K}\left(t=t_{a}\right)=-\frac{e}{m} g^{-1}\left(t_{a}\right) \cdot \nabla A\left(t_{a}\right) \cdot g^{-1}\left(t_{a}\right)
$$

(where

$$
K(t) \equiv K\left(t, t_{a}\right)
$$

and

$$
\left.\mathscr{J}_{t}=-g \nabla_{t}^{2}+\frac{e}{m} F \nabla_{t}+\frac{e}{m} \nabla F \cdot \dot{Q}-\frac{e}{m} \frac{\partial}{\partial t} \nabla A-\frac{e}{m} \nabla \nabla V\right)
$$


it is true that $\omega$, defined by

$$
\omega=g \cdot \dot{K} \cdot N-\tilde{N} \cdot \dot{\tilde{K}} \cdot g
$$

is equal to $\frac{e}{m} F$.

It is simpler to prove that

$$
\Omega \equiv \tilde{K} \cdot \omega \cdot K=\frac{e}{m} \tilde{K} \cdot F \cdot K .
$$

The result for $\omega$ follows then, since $K$ has an inverse. To prove that

$$
\Omega=\frac{e}{m} \tilde{K} \cdot F \cdot K
$$

we show that both sides of the equality satisfy the same first-order differential equation in $t$ with the same boundary conditions at $t_{a}$. We use the facts that $K$ satisfies the Jacobi equation and that hence $\tilde{K}$ satisfies the transpose equation. Now

$$
\Omega \equiv \tilde{K} \cdot \omega \cdot K=\tilde{K} \cdot g \cdot \dot{K}-\tilde{K} \cdot g \cdot K .
$$

Then first,

$$
\begin{aligned}
\Omega\left(t_{a}\right) & =\tilde{K}\left(t_{a}\right) \cdot g\left(t_{a}\right) \cdot \dot{K}\left(t_{a}\right)-\tilde{K}\left(t_{a}\right) \cdot g\left(t_{a}\right) \cdot K\left(t_{a}\right) \\
& =g^{-1} \cdot g \cdot\left(-\frac{e}{m} g^{-1} \cdot \nabla A \cdot g^{-1}\right)-\left(-\frac{e}{m} g^{-1} \cdot \nabla A \cdot g^{-1}\right) \cdot g \cdot g^{-1} \\
& =\frac{e}{m} \tilde{K}\left(t_{a}\right) \cdot F\left(t_{a}\right) \cdot K\left(t_{a}\right)
\end{aligned}
$$

as desired. Next (with dot denoting $\nabla_{t}$ )

$$
\begin{aligned}
\dot{\Omega} & =\dot{\tilde{K}} \cdot g \cdot \dot{K}+\tilde{K} \cdot g \cdot \ddot{K}-\ddot{\tilde{K}} \cdot g \cdot \dot{K}-\dot{\tilde{K}} \cdot g \cdot \dot{K} \\
& =\tilde{K} \cdot g \cdot \ddot{K}-\ddot{\tilde{K}} \cdot g \cdot K .
\end{aligned}
$$

The fact that $K$ is a Jacobi matrix gives

$$
\dot{\Omega}=\frac{e}{m} \dot{\tilde{K}} \cdot F \cdot K+\tilde{K} \cdot F \cdot \dot{K}+\tilde{K} \cdot\left(\nabla F \cdot \dot{Q}-\nabla F \cdot \dot{Q}-\frac{\partial F}{\partial t}\right) \cdot K .
$$

The facts that

$$
\frac{D F}{\partial t}=\frac{\partial F}{\partial t}+\dot{Q} \cdot F
$$

and that

$$
F_{[\alpha \beta ; \mu]}=0
$$

give

$$
\nabla F \cdot \dot{Q}
$$


so that

$$
\begin{aligned}
\dot{\Omega} & =\frac{e}{m}(\tilde{\dot{K}} \cdot F \cdot K+\tilde{K} \cdot F \cdot \dot{K}+\tilde{K} \cdot \dot{F} \cdot K) \\
& =\frac{e}{m}(K \cdot F \cdot K)^{\cdot}
\end{aligned}
$$

as desired.

\section{References}

1. DeWitt-Morette, C.: Feynman's path integral, definition without limiting procedure. Commun. Math. Phys. 28, 47-67 (1972)

2. DeWitt-Morette, C.: Feynman path integrals. I. Linear and affine techniques; II. The FeynmanGreen function. Commun. Math. Phys. 37, 63-81 (1974)

3. DeWitt-Morette, C.: The semi-classical expansion. Ann. Phys. 97, 367-399 (1976)

4. DeWitt-Morette, C., Maheshwari, A., Nelson, B.: Path integration in non-relativistic quantum mechanics. Phys. Rep. 50, 255-372 (1979)

5. Hawking, S.W., Ellis, G.F.R.: The large-scale structure of spacedime. Cambridge, U.K. : Cambridge University Press 1973

6. Sheeks, B.: Some applications of path integration using the prodistribution method. Ph.D. dissertation, University of Texas, Austin 1980

7. Cameron, R.H., Martin, W.T.: Transformation of Wiener integrals under a general class of transformations. Trans. Am. Math. Soc. 58, 184-219 (1945)

8. Nelson, B., Sheeks, B. : Fredholm determinants associated with Wiener integrals. J. Math. Phys. 22, $2132-2136(1981)$

9. DeWitt-Morette, C., Elworthy, K.D., Nelson, B.L., Sammelmann, G.S.: A stochastic scheme for constructing solutions of the Schrödinger equation (Elworthys class. Ann. Inst. Henri Poincare 32, $327-341$ (1980)

Communicated by H. Araki

Received November 16, 1981 